\title{
Acute kidney injury and progression of renal failure after fetal programming in the offspring of diabetic rats
}

Rosana R.M. Corrêa', Karla R.M. Pucci', Laura P. Rocha', Carlos D. Pereira Júnior', Fernanda R. Helmo', Juliana R. Machado', Lenaldo B. Rocha', Aldo R.A. Rodrigues², Maria A. Glória ${ }^{3}$, Camila S.O. Guimarães' ${ }^{1}$ Niels O.S. Câmara ${ }^{3,4}$ and Marlene A. Reis ${ }^{1}$

BACKGROUND: Diseases of adulthood, such as diabetes and hypertension, may be related to changes during pregnancy, particularly in kidney. We hypothesized that acute kidney injury progresses more rapidly in cases of fetal programming. METHODS: Diabetic dams' offspring were divided into: CC (controls, receiving vehicle); DC (diabetics, receiving vehicle); CA (controls receiving folic Acid solution, $250 \mathrm{mg} / \mathrm{kg}$ ); and DA (diabetics receiving folic acid solution). Renal function tests, morphometry, gene, and protein expression of epithelial-mesenchymal transition (EMT) markers were analyzed by qPCR and immunohistochemistry, respectively.

RESULTS: Creatinine, urea, Bowman's space, and EMT markers were increased in CA and DA groups. TGF- $\beta 3$, actin, and fibronectin expression was higher in CA and DA, with significant increase in DA compared to CA 2-mo offspring. There was higher expression level of TGF- $\beta 1$, TGF- $\beta 3$, fibronectin, and vimentin in the offspring of diabetic dams at 5 mo. Increases in TGF- $\beta 1$ and TGF- $\beta 3$ were more evident in the offspring of diabetic dams.

CONCLUSION: Fetal programming promotes remarkable changes in kidney morphology, and function in offspring and renal failure progression may be faster in younger offspring of diabetic dams subjected to an additional injury.

G estational diabetes mellitus can impose several threats both to the mother and to the concept. Diabetic pregnancy increases the risk of intrauterine death, prematurity, perinatal mortality, and congenital malformations (1). It has also been demonstrated by both epidemiological and experimental studies that the offspring of diabetic mothers have an increased risk for the development of cardiovascular disease and insulin resistance in adulthood (2,3). Maternal diabetes promotes remarkable changes in kidney morphology and function and in vascular reactivity in mature offspring (4). In the young animals, an altered response to glucose tolerance test and an inability to excrete a salt overload were also found (5). The inflammation is an important component of progression to chronic renal failure and activation of inflammatory pathways may occur in early stages of diabetic dams offspring development (6).

Acute kidney injury (AKI) is a common disease that affects about $5 \%$ of all hospitalized individuals $(1,7)$. AKI may be resulted from reduced renal perfusion, renal tubules damage related to ischemia, toxicity and obstruction, tubule-interstitial inflammation and edema, or reduced filtration capacity of the glomerulus (8). The overexpression of epithelial-mesenchymal transition (EMT) markers has been described as an indicator of the disease progress (9). Presently, it has been observed a considerable demographic change in the AKI-affected population. There is an increase in the age of patients and association with comorbidities such as diabetes, hypertension, myocardial revascularization surgeries, and sepsis are increasingly common. Many of these changes are possibly related to the advent of dialysis (8).

Fetal programming is a term used to designate alterations in fetal gene expression due to exposure to certain adverse events during pregnancy like maternal obesity or diabetes. These events may lead to modifications in the metabolism and mechanisms of growth of the offspring, resulting in an increase of susceptibility to chronic disease in later life (10). Several studies have shown an association between diseases of adulthood, such as diabetes and hypertension, and changes during pregnancy, particularly in the kidney $(1,3,11,12)$. However, no studies have correlated the influence of intrauterine environment changes on the development of new lesions acquired during adulthood, such as AKI. Therefore, we hypothesized that AKI progresses more rapidly when associated with a higher intensity of renal lesions and the increased expression of EMT markers that may be caused during fetal programming. Some studies demonstrate that maternal diabetes induced by STZ may be associated with early renal consequences in the offspring. Glomerular hypertrophy, decrease in glomerular filtration rate and renal plasma flow, increase in blood pressure and changes in vascular response related to nitric oxide were observed. These studies suggest that fetal programming may cause impaired renal function $(1,3)$.

'General Pathology Discipline, Department of Genetics, Ecology and General Pathology. Biological and Natural Sciences Institute, Triângulo Mineiro Federal University, Uberaba, Brazil; ${ }^{2}$ Discipline of Physiology, Department of Biochemistry, Molecular Biology, Pharmacology, Physiology and Chemical, Biological and Natural Sciences Institute, Triângulo Mineiro Federal University, Uberaba, Brazil; ${ }^{3}$ Nephrology Division, Department of Medicine, Federal University of São Paulo (UNIFESP), São Paulo, Brazil; ${ }^{4}$ Department of Immunology, Institute of Biomedical Sciences, University of São Paulo (USP), São Paulo, Brazil. Correspondence: Rosana R.M. Corrêa (rosana@patge.uftm.edu.br) 


\section{RESULTS}

Effects of Maternal Diabetes and Folic Acid on Blood Pressure, Heart Rate, Body Weight, Lee Index, Number of Glomeruli,

\section{Creatinine, and Urea}

There was no significant difference in blood pressure, heart rate, body weight, number of glomeruli, and Lee index between the groups at 2 and 5 mo old. A significant increase of creatinine and urea levels was observed in the offspring of the controls and diabetic dams who received folic acid at 2 and 5 mo (Figures 1a,b and 2a,b).

\section{Effects of Maternal Diabetes and Folic Acid on the Expression of Transforming Growth Factor TGF- $\beta 1$, TGF- $\beta 3$, Actin, Fibronectin, and Vimentin and the Percentage of Renal Fibrosis}

There was no significant difference in the expression of TGF$\beta 1$, TGF- $\beta 3$, actin, fibronectin, and vimentin in the offspring of diabetic dams compared to that of controls at $2 \mathrm{mo}$, DC vs. CC. Folic acid administration increased the expression of these markers in both diabetic and control offspring at 2 mo. However, significant differences were observed only between CC and CA when TGF- $\beta 1$ was analyzed, between DC and DA when TGF- $\beta 3$ was analyzed and between DC and DA when actin was analyzed (Figure 1c-e). The increases in TGF- $\beta 3$ and actin were significantly higher in the offspring of diabetic dams who received folic acid compared to control offspring who received folic and offspring of diabetic dams who did not received folic acid at $2 \mathrm{mo}$, DA vs. CA and DC (Figure 1d,e). The same relationship was observed with fibronectin, however, without significant difference (Figure 1f). There was a higher expression level of TGF- $\beta 1$, TGF- $\beta 3$, fibronectin, and vimentin in the offspring of diabetic dams at $5 \mathrm{mo}$, DC vs. CC. Nevertheless, our results demonstrated significant difference only when vimentin was analyzed (Figure $2 \mathrm{~g}$ ). In the offspring of diabetic dams as well as in controls, there was an increase in all markers observed when the offspring received folic acid, CA vs. CC and DA vs. DC (Figure $2 \mathrm{c}-\mathrm{g}$ ). The increases in TGF$\beta 1$ and TGF- $\beta 3$ were more evident in the offspring of diabetic dams, DA vs. CA (Figures $2 \mathrm{c}$,d). There was no significant difference in the extent of renal fibrosis in any comparison.

Correlation Between Markers of Renal Function and TGF- $\beta 1$, TGF$\beta 3$, Actin, Fibronectin, Vimentin, and Renal Fibrosis

Significant positive correlations between urea and TGF$\beta 1$ (rS: 0.532; $P<0.001$ ), urea and TGF- $\beta 3$ (rS: 0.641; $P<0.001)$, urea and actin (rS: $0.781 ; P<0.001)$, urea and fibronectin $(\mathrm{rS}: 0.505 ; P<0.002)$, urea and vimentin $(\mathrm{rS}$ : $0.555 ; P<0.001)$, creatinine and TGF- $\beta 1$ (rS: $0.420 ; P<$ $0.012)$, creatinine and TGF- $\beta 3$ (rS: $0.561 ; P<0.001)$, creatinine and actin (rS: 0.739; $P<0.001)$, creatinine and fibronectin $(\mathrm{rS}: 0.532 ; P<0.001)$ and creatinine and vimentin $(\mathrm{rS}$ : $0.472 ; P<0.003)$ were observed. There was no significant difference in renal fibrosis in any of the analyses.

\section{DISCUSSION}

No significant differences in blood pressure, heart rate, body weight, and Lee index were observed between the offspring of diabetic dams and controls, suggesting normal development of the evaluated cases, in agreement with previous data $(1,12)$.

Significant increases in creatinine and urea levels were found in the offspring of diabetic progenitors and controls who received folic acid at 2 and 5 mo. High doses of folic acid cause decreased renal function, as demonstrated by increased serum creatinine levels above $150 \%$ and blood urea nitrogen levels above $200 \%$, both compared to controls. Kidney injury caused by folic acid is induced either by the deposition of folic acid crystals, which block the tubules, or by direct toxicity of tubular epithelial cells (13). This process is characterized by tubular necrosis in the acute phase, followed by epithelial regeneration, induction of inflammatory cytokines such as tumor necrosis factor, invasion of monocytes/macrophages (14), development of interstitial fibrosis in the chronic phase (13), loss of peritubular capillaries, atrophy, and tissue hypoxia (14). Shortly after administration, folic acid induces a rapid appearance of crystals in the renal tubules (15), with renal insufficiency confirmed by the decline of renal function $24 \mathrm{~h}$ after administration (14). Our model evaluated renal changes $36 \mathrm{~h}$ after the administration of folic acid, and the injury induced up to this timepoint was sufficient to cause a decline in renal function.

The expression of TGF- $\beta 1$, TGF- $\beta 3$, actin, fibronectin, and vimentin displayed a significant positive correlation with the levels of urea and creatinine, and there was no significant difference in renal fibrosis in any of the analyses. The tubule interstitial space plays an important role in the progression of chronic kidney diseases (9). Changes in this compartment, such as tubular degeneration and cellular infiltration, are common in kidney diseases that progress to renal failure (16). The new cells in the interstitium release proinflammatory cytokines, such as tumor necrosis factor- $\alpha$, interleukin (IL)-6, and IL-1 $\beta$, which activate resident fibroblasts and lead tubular epithelial cells to begin the process of EMT, in which epithelial cells undergo biochemical alterations and assume a mesenchymal phenotype (6). EMT is associated with release of fibroproliferative molecules, such as TGF- $\beta$ and PAI- 1 , and with the production of extracellular matrix proteins, such as fibronectin and collagen types I and III $(17,18)$. In our model, even in the absence of significant differences in fibrosis, we demonstrated that prefibrogenic markers involved in EMT correlated positively with urea and creatinine levels in an experimental model of the short-term induction of renal failure. These data are consistent with other studies (4), demonstrating that these can be used as markers of AKI to predict its evolution, thereby increasing the chances of more effective interventions in treating renal diseases.

There was no significant difference in the expression of TGF- $\beta 1$, TGF- $\beta 3$, actin, fibronectin, and vimentin in the offspring of diabetic dams as compared to that of controls (DC2 X CC2). Another research group examined the activation of inflammatory pathways in the kidneys of 2-mo-old animals from diabetic progenitors. They demonstrated that the expression of inflammatory cytokines could be triggered early in the life of the offspring, at the moment when renal injury had not been detected by clinical parameters (19). A study, that used a 
a

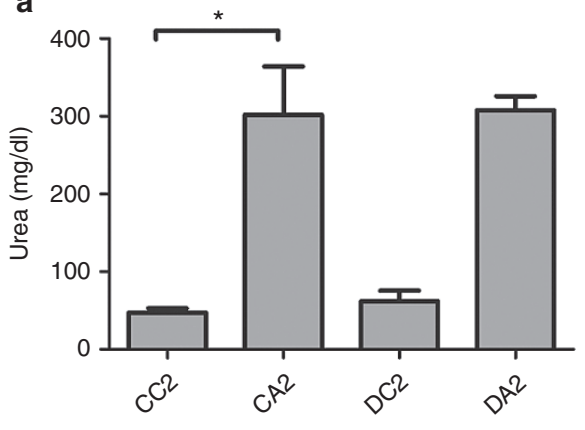

C
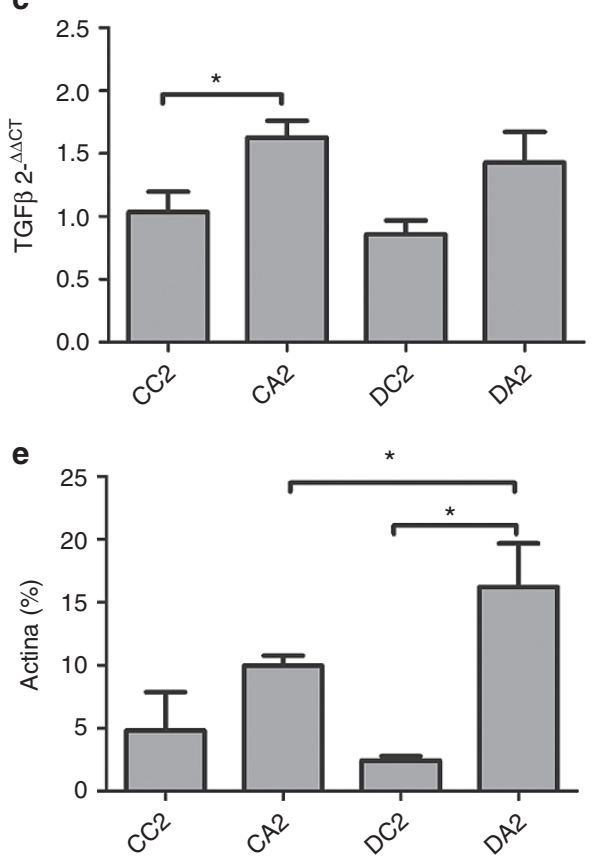

g

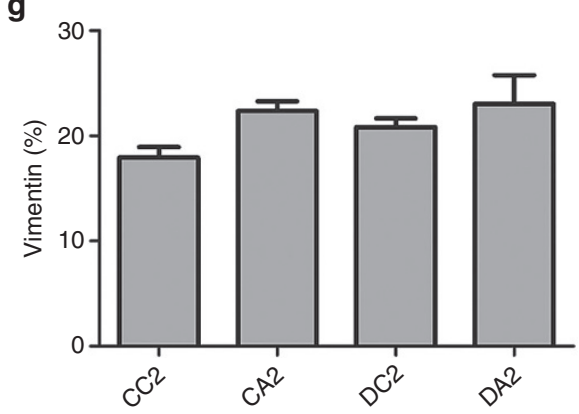

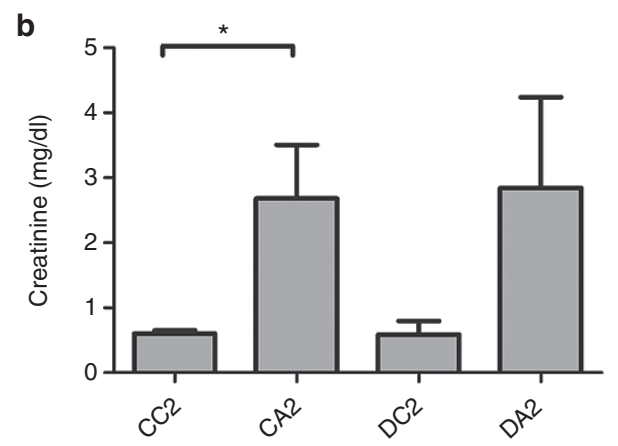

d

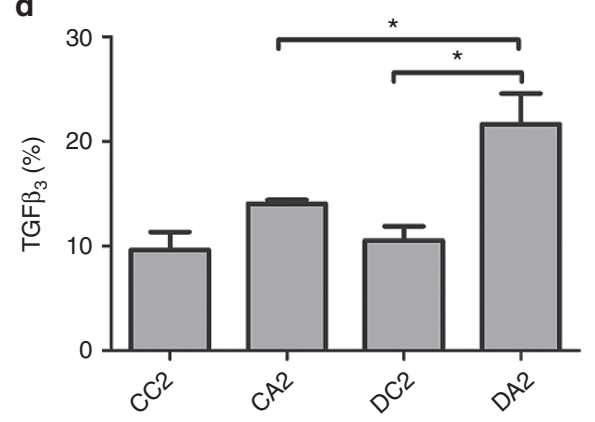

$\mathbf{f}$

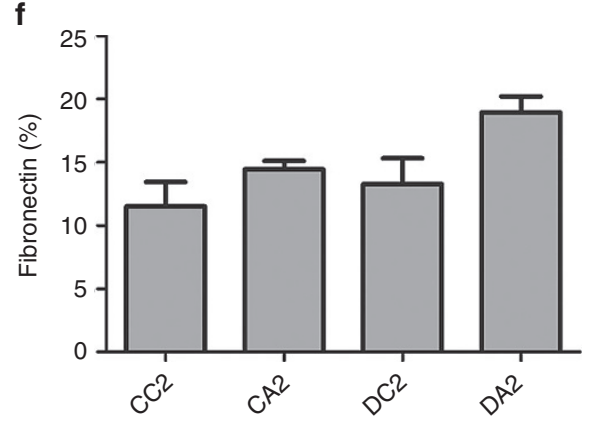

Figure 1. Analysis of renal function, glomerular number, urinary space, and epithelium mesenchymal transition (EMT) markers in the offspring at 2 mo old. Data from control and diabetic offspring with or without folic acid treatment. Control offspring $(\mathrm{CC} 2, n=5), C$ plus folic acid (CA2, $n=5)$, diabetic offspring (DC2, $n=5)$ and diabetic plus Folic acid (DA2, $n=5)$. Number after the letter indicates the age of the animals, in months. *Significance level, $P<0.05$.

similar methodology that we used, described the increase in cytokines in the offspring at 2 mo from diabetic dams induced by streptozotocin (20). However, our data did not demonstrate changes in markers of EMT in the absence of lesions in animals with 2 mo. If these inflammatory pathways occurred in the offspring of our experiment, probably it does not affect markers of EMT in the absence of injury.

There was a significant higher expression of vimentin in the offspring of diabetic dams at 5 mo, however TGF- $\beta 1$, TGF- $\beta 3$, and fibronectin were also increased in this group (DC5 > CC5). Our model showed no significant differences in parameters commonly described to assess fetal programming, including the number of glomeruli and glomerular compensatory hypertrophy. One study showed that these changes could be observed long-term in the diabetic offspring at $12 \mathrm{mo}$, concluding that diabetes is an important inducer of fetal programming, which accelerates renal aging by promoting increased nephron loss (3). The lack of consensus on the presentation of these changes may 
a

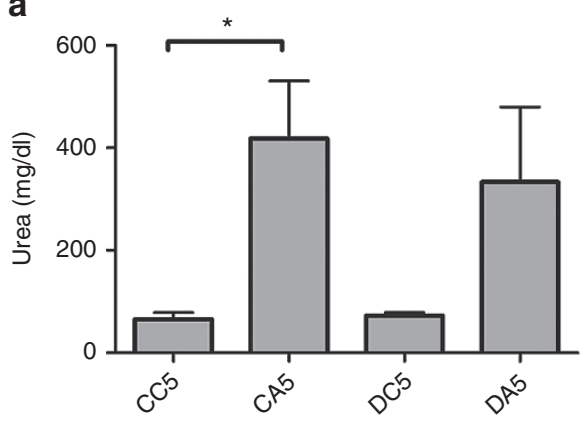

C

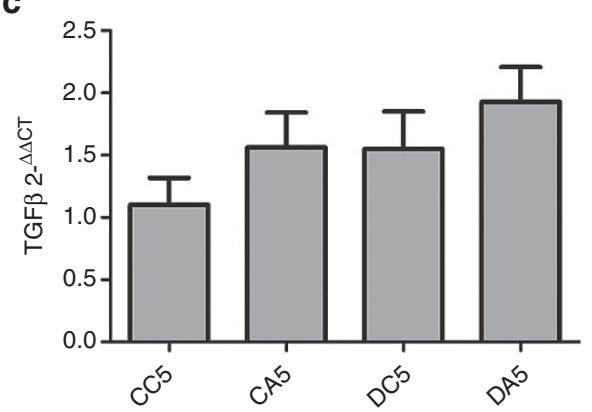

e

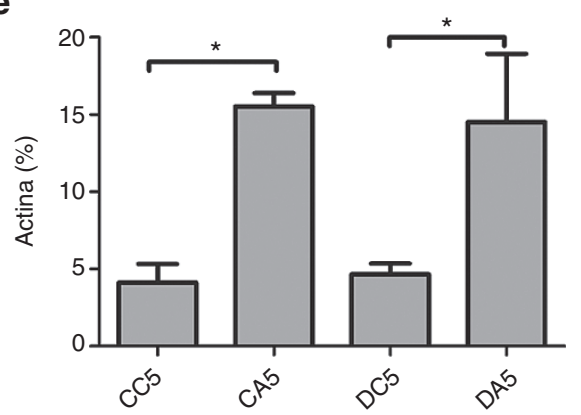

g

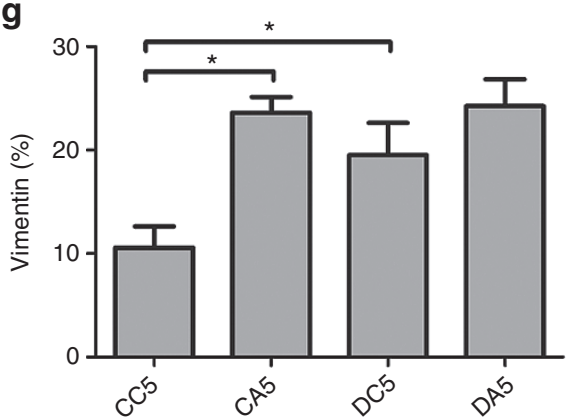

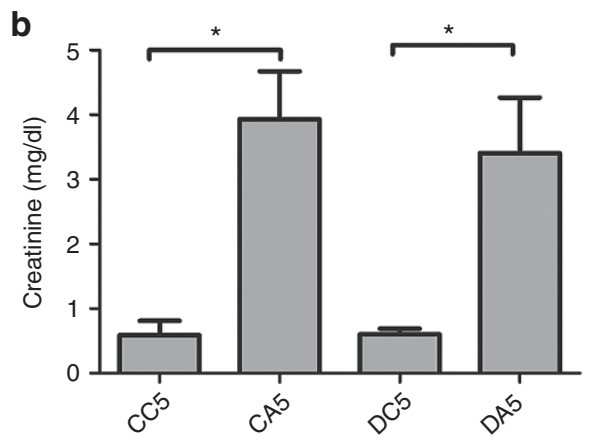

d

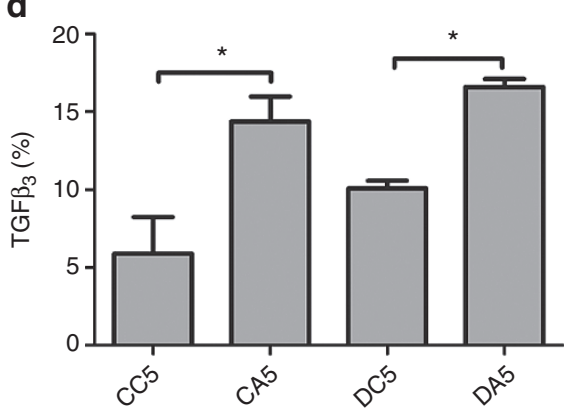

f

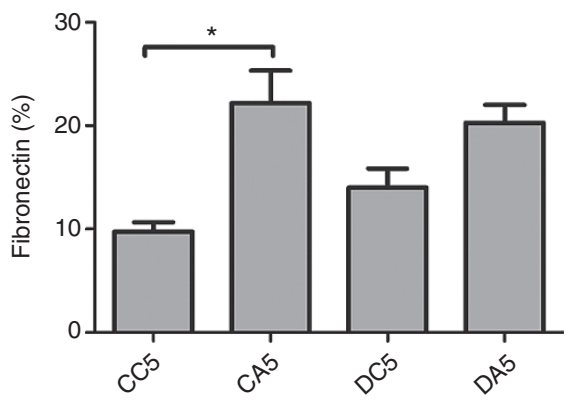

Figure 2. Analysis of renal function, glomerular number, urinary space, and epithelium mesenchymal transition (EMT) markers in the offspring at 5 mo old. Data from control and diabetic offspring with or without folic acid treatment. Control offspring (CC5, $n=5), C$ plus folic acid (CA5, $n=5)$, diabetic offspring (DC5, $n=5)$ and diabetic plus folic acid (DA5, $n=5)$. Number after the letter indicates the age of the animals, in months. ${ }^{* S i g n i f i c a n c e ~ l e v e l, ~}$ $P<0.05$.

be related to the different models of diabetes during pregnancy, as different doses, routes of administration, and timepoints (before or during pregnancy) were chosen for STZ administration.

Our model showed increased EMT markers in diabetic offspring at $5 \mathrm{mo}$. EMT has been described in pathological conditions, such as severe fibrosis and neoplastic invasion and metastasis. After a stimulus that increases TGF- $\beta$ production, the mesenchymal phenotype is induced, characterized by the expression of cytoskeleton cellular proteins, such as vimentin, and an increased deposition of extracellular matrix proteins, such as collagen and fibronectin (21). Our results suggest that renal changes in diabetic offspring are more evident in offspring at 5 mo when they were compared with offspring at 2 mo and this experiment suggests that increased EMT markers may be new information to support the theory of fetal programming. 
In a study which investigated possible effects of maternal diabetes on the offspring at different ages, it was observed that in older rats, the inflammatory mechanism persists and other inflammatory cytokines are detected, suggesting that aging may amplify this process (3). There is an increased influx of inflammatory cells, alteration of cell functions, reduction in the function of stem and progenitor cells, and an age-dependent increase in the expression of TGF- $\beta 1$ in the kidney (21). Thus, the aging mechanism itself is able to activate the expression of TGF- $\beta$ and its isoforms. Despite TGF- $\beta 3$ may work in cooperation with TGF- $\beta 1$ during EMT induction, presenting an important role in this process (22), some studies suggest that TGF- $\beta 3$ may antagonize the profibrotic effects of TGF- $\beta 1$ (23) and is most related to tissue repair function, contributing to the restoration of normal function after a lesion (24). As our data also showed an increase in TGF- $\beta 3$ expression, it was demonstrated an increase in other mediators of EMT, indicating a favorable environment for reparative processes in diabetic offspring at older ages.

Actin expression did not differ in relation to fetal programming (DC5 vs. CC5). One study evaluated the expression of $\alpha$-smooth muscle actin (SMA) in the glomeruli, renal tubules, and renal interstitium, usually observed during development and in pathological conditions, and found no changes in its expression in diabetic offspring until 1 mo of age (1). No studies demonstrated increased actin expression in diabetic offspring at ages above our oldest age group, 5 mo. Thus, we conclude that actin expression does not change in the offspring of diabetic dams up to this age. Further studies are needed to evaluate its expression at older ages to confirm the effect of fetal programming on this marker.

Folic acid administration increases the expression of these markers in both diabetic and control offspring at 2 and 5 mo. The increase in TGF- $\beta 3$ and actin was significantly higher in the offspring of diabetic dams treated with folic acid (DA2 > CA2). Although not significant, a similar relationship was observed with fibronectin. In our studies, the changes between groups DA and CA show that programming may worsens the evolution of renal impairment, evidenced by the expression of these markers at 2 mo. TGF- $\beta$ induces the expression of $\alpha$-SMA in a variety of mesenchymal cells in culture and is often associated with the phenotypic change of fibroblasts into myofibroblasts in kidney lesions (25). In another study, cultured mice podocytes underwent phenotypic changes with increased expression of $\alpha$-SMA when subjected to the influence of TGF- $\beta 1$ (26), an immunomodulatory cytokine that is important in the human kidney (27). Our data demonstrate modification of the kidney response to damage in reprogrammed cases, clearly observed in 2 mo group.

It was not observed significant changes in EMT inducers and markers in the offspring of $5 \mathrm{mo}$. Aging is associated with a loss of renal mass, which has little influence on renal function but may increase vulnerability to other lesions of the kidney (28). Furthermore, some authors suggest that aging is associated with an inflammatory status mediated by TGF- $\beta(29,30)$. As described previously, maternal diabetes promotes the increase of EMT markers in diabetic offspring. However, in our model, the presence of injury in the offspring of $5 \mathrm{mo}$, showed that the programming does not influence the progression of renal failure. Thus, faced with kidney injury, reprogrammed young individuals may exhibit more pronounced lesion progression than older individuals, demonstrating that in older age groups, the incidence of disease is not influenced by programming, which could suggest that fetal programming may be transient.

Although there are many doubts concerning the role of EMT in renal fibrosis in vivo and that more studies are still needed to support the EMT theory $(31,32)$, we strongly suggest that there is an increase of EMT mediators in older diabetic offspring, representing another parameter for assessing fetal programming. Our data also demonstrate modification of the pattern of the response of the kidney in relation to damage in reprogrammed subjects. Thus, the mediators increase at younger ages, leading to more rapid progression of kidney injury.

\section{METHODS}

All procedures used in this study were approved and performed in accordance with guidelines of the Ethics Committee of Biomedical Institute, Triângulo Mineiro Federal University (Permit number: 168) and were conducted following the Guide for Care and Use of Laboratory Animals published by the United States National Institutes of Health (Publication No.85-23, revised 1996). Wistar rats from our colony were maintained in a room at $22 \pm 1{ }^{\circ} \mathrm{C}$ with a $12 \mathrm{~h}$ light cycle and $60 \%$ humidity, and all efforts were made to minimize suffering.

\section{Animal Model}

Diabetes mellitus was induced by streptozotocyn, $(50 \mathrm{mg} / \mathrm{kg})$, given by a single intraperitoneal injection to female Wistar rats (250-300 g). Control animals were given an equivalent amount of citrate buffer. Diabetic state was confirmed $48 \mathrm{~h}$ after, by measuring blood glucose. Only those animals with glycemic levels above $250 \mathrm{mg} / \mathrm{dl}$ were considered for mating. After 3-7 d of diabetic state confirmation, the rats were caged overnight with a male. Vaginal smears were taken in the following morning and a positive smear was considered as day 0 of gestation. All dams were housed and fed individually with the same diet. After birth, each litter, consisting of around six male rats, was left with the mother for $28 \mathrm{~d}$; if the male number was not enough to complete 6 , females were used but discarded at weaning, according to previous studies (1). At 2 and 5 mo, the offspring were divided into four subgroups: group CC (offspring of controls dams, receiving $\mathrm{NaHCO} 3$ - vehicle); group DC (offspring of diabetic dams, receiving vehicle); group CA (offspring of controls dams, receiving folic acid) and group DA (offspring of diabetic dams, receiving folic acid). In each age analyzed (groups at 2 and 5 mo old), we used six dams. Each dam obtained an average of at least 4 males per litter, being selected 24 male pups from the six dams. Each pup received an identification number in accordance with its dam, and these were randomly divided among the four groups, in a way that each group was composed of pups from six different dams. The folic acid dose was $250 \mathrm{mg} / \mathrm{kg}$ in $0.2 \mathrm{ml}$ of $300 \mathrm{mmol} / \mathrm{l}$, similar to that used by Gupta et al. (13), given by a single intraperitoneal injection. Euthanasia was performed $36 \mathrm{~h}$ after application of folic acid by excessive anesthetic dose.

\section{Lee Index}

Lee Index for each offspring obtained the obesity parameter. This index was calculated by the cube root of body weight $(\mathrm{g}) \times 10$ /nasoanal length $(\mathrm{mm})$, for which a value equal to or lower than 0.300 was classified as normal at month 2 and 5 of life. For values higher than 0.300 , the rats were classified as obese (33).

\section{Blood Pressure}

Measurement of systemic blood pressure was performed by tail cuff plethysmography after $24 \mathrm{~h}$ of induction of renal failure in the offspring. 


\section{Renal Function}

To carry out renal function studies, the serum was prepared after collecting the blood samples through the cardiac puncture of the animal following overnight fasting, at the time of euthanasia. Renal function was assessed by measuring the levels of creatinine and blood urea nitrogen (BUN) using a commercially available kit (LABTEST, Belo Horizonte, Brasil). The level of serum creatinine and BUN was expressed as milligrams per $100 \mathrm{ml}(\mathrm{mg} / \mathrm{dl})$.

\section{Morphometric Study}

Morphometry was performed with longitudinal sections of the right kidney sectioned along the height of the hilum. The slides were stained with hematoxylin and eosin, and we used an interactive image analyzing system from Leica Microsystems, Leica QWin (Wetzlar, Germany). The number of glomeruli was evaluated in the entire renal cortex, at an amplification of $10 \times$. The areas of the Bowman's capsule and capillary tuft of the glomeruli located in the juxtamedullar, intermediate, and superficial zones were analyzed at an original amplification of $620 \times$ magnification and expressed in $\mu \mathrm{m}^{2}$. Morphometry was performed on the urinary pole and/or vascular pole glomeruli. The area of the Bowman's space was determined by the difference between the area of the glomerular capillary tuft and the area of Bowman's capsule. After the hydration of the 5- $\mu \mathrm{m}$ sections, the slides were stained for $1 \mathrm{~h}$ in a $0.1 \%$ Sirius Red solution (Direct Red 80, Aldrich, diluted in saturated picric acid solution), followed by rapid washing in running tap water and counterstained with Harris's hematoxylin for $15 \mathrm{~min}$, dehydrated and mounted in synthetic resin. Picrosirius-stained sections were evaluated by ordinary polychromatic and polarized light microscopies, and collagen fibers were quantified.

\section{TGF- $\beta$ Expression}

Kidney samples were quickly frozen in liquid nitrogen. Total RNA was isolated from kidney tissue using TRizol Reagent (Invitrogen, Carlsbad, CA) methodology and RNA concentration was determined by spectrophotometer readings at an absorbance of $260 \mathrm{~nm}$. Firststrand cDNA were synthesized using the MML-V reverse transcriptase (Promega, Madison, WI). All experimental protocols of real-time PCR were based on the manufacturer's recommendation using the TaqMan gold RT-PCR Core Reagents Kit (Applied Biosystems, Foster City, CA). Primer and probe to TGF- $\beta$ (Rn00572010_m1) was purchased from Applied Biosystems (TaqMan Gene Expression Assays). Cycling conditions were as follows: $10 \mathrm{~min}$ at $95^{\circ} \mathrm{C}$, followed by 45 cycles of $15 \mathrm{~s}$ at $95^{\circ} \mathrm{C}$ and $1 \mathrm{~min}$ at $60^{\circ} \mathrm{C}$. The amount of the target gene was normalized first to an endogenous reference Hypoxanthine Phosphoribosyltransferase (HPRT) and then relative to a calibrator (sample with the lowest expression, namely, sham-operated animals), using the 2-DDCt method. Hence, steady-state mRNA levels were expressed as an $n$-fold difference relative to the calibrator. Analyses were performed with the Sequence Detection Software 1.9 (SDS).

\section{Immunohistochemical}

We used the rabbit antibody to Smooth Muscle Actin (1:50, DakoCytomation, Glostrup, Dinamarca); Vimentin (1:100, DakoCytomation); TGFß3 (1:40, Diagnostic Biosystems, Pleasanton, CA); e Fibronectin (1:120, Diagnostic Biosystems). Immunohistochemistry was performed on formaldehyde-fixed and paraffin-embedded (sections: $2 \mu \mathrm{m}$ thick) from right kidney. Analysis were performed using a light microscope and the software Leica QWin.

\section{Statistical Analysis}

All data were described as mean \pm SEM. Statistical evaluation of the data was carried out using the $t$-test or the one-way ANOVA followed by Tukey's post-test, or Kruskal-Wallis test followed by Dunn post-test. The correlation between two variables with not normal distribution was analyzed by Spearman (rS) tests. A value lower than 0.05 was considered to be significant. All statistical analyses were performed with the aid of GraphPad Prism 5.0 (GraphPad Software, San Diego, CA).

\section{STATEMENT OF FINANCIAL SUPPORT}

This work was financially supported by Conselho Nacional de Desenvolvimento Científico e Tecnológico (CNPq, Brasil), Coordenação de
Aperfeiçoamento de Pessoal de Nível Superior (CAPES, Brasil), Fundação de Amparo à Pesquisa do Estado de Minas Gerais (FAPEMIG, Brasil) and Fundação de Ensino e Pesquisa de Uberaba (FUNEPU, Brasil).

Disclosures: All authors declare that they had no potential conflicts of interest relevant to this article.

\section{REFERENCES}

1. Magaton A, Gil FZ, Casarini DE, Cavanal Mde F, Gomes GN. Maternal diabetes mellitus-early consequences for the offspring. Pediatr Nephrol 2007;22:37-43.

2. Van Assche FA, Holemans K, Aerts L. Long-term consequences for offspring of diabetes during pregnancy. Br Med Bull 2001;60:173-82.

3. Rocha SO, Gomes GN, Forti AL, et al. Long-term effects of maternal diabetes on vascular reactivity and renal function in rat male offspring. Pediatr Res 2005;58:1274-9.

4. Kanwar YS, Nayak B, Lin S, et al. Hyperglycemia: its imminent effects on mammalian nephrogenesis. Pediatr Nephrol 2005;20:858-66.

5. Moritz KM, Dodic M, Wintour EM. Kidney development and the fetal programming of adult disease. Bioessays 2003;25:212-20.

6. Correa-Costa M, Semedo P, Monteiro AP, et al. Induction of heme oxygenase- 1 can halt and even reverse renal tubule-interstitial fibrosis. PLoS One 2010;5:e14298.

7. Paller MS. Acute renal failure: controversies, clinical trials, and future directions. Semin Nephrol 1998;18:482-9.

8. Thadhani R, Pascual M, Bonventre JV. Acute renal failure. N Engl J Med 1996;334:1448-60.

9. Strutz F, Neilson EG. New insights into mechanisms of fibrosis in immune renal injury. Springer Semin Immunopathol 2003;24:459-76.

10. Capra L, Tezza G, Mazzei F, Boner AL. The origins of health and disease: the influence of maternal diseases and lifestyle during gestation. Ital J Pediatr 2013;39:7.

11. Wichi RB, Souza SB, Casarini DE, Morris M, Barreto-Chaves ML, Irigoyen MC. Increased blood pressure in the offspring of diabetic mothers. Am J Physiol Regul Integr Comp Physiol 2005;288: R1129-33.

12. Rocco L, Gil FZ, da Fonseca Pletiskaitz TM, de Fátima Cavanal M, Gomes GN. Effect of sodium overload on renal function of offspring from diabetic mothers. Pediatr Nephrol 2008;23:2053-60.

13. Gupta A, Puri V, Sharma R, Puri S. Folic acid induces acute renal failure (ARF) by enhancing renal prooxidant state. Exp Toxicol Pathol 2012;64:225-32

14. Long DA, Woolf AS, Suda T, Yuan HT. Increased renal angiopoietin-1 expression in folic acid-induced nephrotoxicity in mice. J Am Soc Nephrol 2001;12:2721-31.

15. Koziolek MJ, Müller GA, Zapf A, et al. Role of CX3C-chemokine CX3C$\mathrm{L} /$ fractalkine expression in a model of slowly progressive renal failure. Nephrol Dial Transplant 2010;25:684-98.

16. Nath KA. Tubulointerstitial changes as a major determinant in the progression of renal damage. Am J Kidney Dis 1992;20:1-17.

17. Bombara MP, Webb DL, Conrad P, et al. Cell contact between T cells and synovial fibroblasts causes induction of adhesion molecules and cytokines. J Leukoc Biol 1993;54:399-406.

18. Burger D, Rezzonico R, Li JM, et al. Imbalance between interstitial collagenase and tissue inhibitor of metalloproteinases 1 in synoviocytes and fibroblasts upon direct contact with stimulated T lymphocytes: involvement of membrane-associated cytokines. Arthritis Rheum 1998;41:1748-59.

19. Babazono T, Hanai K, Suzuki K, et al. Lower haemoglobin level and subsequent decline in kidney function in type 2 diabetic adults without clinical albuminuria. Diabetologia 2006;49:1387-93.

20. Correa-Costa M, Landgraf MA, Cavanal MF, et al. Inflammatory milieu as an early marker of kidney injury in offspring rats from diabetic mothers. Eur J Pharmacol 2012;689:233-40.

21. Xu J, Lamouille S, Derynck R. TGF-beta-induced epithelial to mesenchymal transition. Cell Res 2009;19:156-72.

22. Medici D, Hay ED, Olsen BR. Snail and Slug promote epithelial-mesenchymal transition through beta-catenin-T-cell factor-4-dependent expression of transforming growth factor-beta3. Mol Biol Cell 2008;19:4875-87. 


\section{Articles $\mid$ Corrêa et al.}

23. Shah M, Foreman DM, Ferguson MW. Neutralisation of TGF-beta 1 and TGF-beta 2 or exogenous addition of TGF-beta 3 to cutaneous rat wounds reduces scarring. J Cell Sci 1995;108 (Pt 3):985-1002.

24. Buckley S, Bui KC, Hussain M, Warburton D. Dynamics of TGF-beta 3 peptide activity during rat alveolar epithelial cell proliferative recovery from acute hyperoxia. Am J Physiol 1996;271(1 Pt 1):L54-60.

25. Barnes VL, Musa J, Mitchell RJ, Barnes JL. Expression of embryonic fibronectin isoform EIIIA parallels alpha-smooth muscle actin in maturing and diseased kidney. J Histochem Cytochem 1999;47:787-98.

26. Chen CA, Hwang JC, Guh JY, Tsai JC, Chen HC. TGF-betal and integrin synergistically facilitate the differentiation of rat podocytes by increasing alpha-smooth muscle actin expression. Transl Res 2006;148:134-41.

27. Qi W, Chen X, Polhill TS, et al. TGF-betal induces IL-8 and MCP-1 through a connective tissue growth factor-independent pathway. Am J Physiol Renal Physiol 2006;290:F703-9.
28. Melk A, Halloran PF. Cell senescence and its implications for nephrology. J Am Soc Nephrol 2001;12:385-93.

29. Cufí S, Vazquez-Martin A, Oliveras-Ferraros C, Martin-Castillo B, Joven J, Menendez JA. Metformin against TGF $\beta$-induced epithelial-to-mesenchymal transition (EMT): from cancer stem cells to aging-associated fibrosis. Cell Cycle 2010;9:4461-8.

30. Franceschi C. Inflammaging as a major characteristic of old people: can it be prevented or cured? Nutr Rev 2007;65(12 Pt 2):S173-6.

31. Kriz W, Kaissling B, Le Hir M. Epithelial-mesenchymal transition (EMT) in kidney fibrosis: fact or fantasy? J Clin Invest 2011;121:468-74.

32. Liu Y. New insights into epithelial-mesenchymal transition in kidney fibrosis. J Am Soc Nephrol 2010;21:212-22.

33. Bernardis LL, Patterson BD. Correlation between 'Lee index' and carcass fat content in weanling and adult female rats with hypothalamic lesions. J Endocrinol 1968;40:527-8. 